\title{
MDC and BLC are independently associated with the significant risk of early stage lung adenocarcinoma
}

\author{
Yanwei Zhang ${ }^{1}$, Keke $\mathrm{Yu}^{2}$, Song Hu${ }^{3}$, Yuqing Lou ${ }^{1}$, Chunxing Liu ${ }^{4}$, Jianlin $\mathrm{Xu}^{1}$, Rong \\ $\mathbf{L i}^{1}$, Xueyan Zhang ${ }^{1}$, Huimin Wang ${ }^{1}$, Baohui Han ${ }^{1}$ \\ ${ }^{1}$ Department of Pulmonary Medicine, Shanghai Chest Hospital, Shanghai Jiao Tong University, Shanghai, PR China \\ ${ }^{2}$ Department of Biobank, Shanghai Chest Hospital, Shanghai Jiao Tong University, Shanghai, PR China \\ ${ }^{3}$ Department of Research Center, Shanghai Chest Hospital, Shanghai Jiao Tong University, Shanghai, PR China \\ ${ }^{4}$ Department of Laboratory Medicine, Huadong Sanatorium, Wuxi, Jiangsu Province, PR China \\ Correspondence to: Baohvi Han, email: xkyyhan@gmail.com \\ Keywords: early stage lung adenocarcinoma, subcentimeter lung adenocarcinoma, inflammatory biomarkers \\ Received: August 17, $2016 \quad$ Accepted: October 05, 2016 Published: November 03, 2016
}

\section{ABSTRACT}

Background: This prospective study was designed to investigate the association between ten circulating inflammatory biomarkers and the risk for early stage lung adenocarcinoma. Methods: All inflammatory biomarkers were measured in 228 patients with early stage (IA to IIB) lung adenocarcinoma and 228 age-, sex- and smoking-matched healthy controls by using the Luminex bead-based assay. Results: Only two biomarkers were significantly associated with the risk of early stage lung adenocarcinoma after the Bonferroni correction: the multivariate odd ratio (OR) (95\% confidence interval or $\mathrm{CI})$ was $0.29(0.16-0.53)$ for MDC and 4.17 (2.23-7.79) for BLC for the comparison of patients in the $4^{\text {th }}$ quartile with the $1^{\text {st }}$ quartile (both $P<0.0001$ ). When analysis was restricted to never smokers (196 patients/196 controls), MDC and BLC were still significantly associated with the risk of early stage lung adenocarcinoma (OR, 95\% CI, P: 0.37, 0.21-0.66, $P<0.0001$ for $M D C$ and 2.78, 1.48-5.22, $P=0.001$ for BLC). Furthermore, elevated BLC was associated with a 2.90-fold (95\% CI: 1.03-8.17, $P=0.037$ ) increased risk of subcentimeter lung adenocarcinoma, and there was an increasing trend for BLC with the progression of subcentimeter lung adenocarcinoma. Conclusion: Our findings demonstrated that MDC and BLC were independently associated with the significant risk of early stage lung adenocarcinoma, even in nonsmokers and in stage IA patients. BLC was further identified to play a carcinogenic role in the progression of lung adenocarcinoma.

\section{INTRODUCTION}

Lung cancer is the leading cause of cancer-related death worldwide. As an aggressive histopathologic type of lung cancer, lung adenocarcinoma has recently aroused extensive concerns of scientific community $[1,2]$. The dismal 5-year survival rate of lung cancer is mainly due to late-stage diagnosis for the majority of patients. In fact, the stage of lung cancer has a major impact on survival rate, as up to $65 \%$ of patients diagnosed with early stage lung cancer survived five years compared to less than $10 \%$ of those entering an advanced stage at diagnosis [3, 4]. Detecting lung adenocarcinoma at an early stage is thus vital to improve the prognosis and prolong the survival in clinical practice.
There is compelling evidence in medical literature for the diagnostic utility of low-dose computed tomography (LDCT) in patients with early stage lung cancer. A national lung screening trial or NLST conducted in 2011 has showed that LDCT screening can reduce lung cancer mortality by $20 \%$ [5]. As recommended by the United States Preventive Services Task Force guidelines, it is necessary to implement annual LDCT screening for individuals at high risk for lung cancer [6]. Using LDCT screening, the detection rate of patients with stage I lung cancer was $70 \%$, which was exceedingly higher than that of $16 \%$ under routine care [7], which highlights the importance of LDCT as a practical diagnostic tool for lung cancer. However, a major problem facing global researchers currently is the high false-positive rate of 
LDCT screening, as recorded in the NLST study: nearly $96 \%$ of abnormal results were false-positive, which resulted in unnecessary subsequent diagnostic screening procedures and even complications from invasive steps [5]. It is therefore of timely importance to identify biomarkers to facilitate the diagnostic utility of LDCT during lung cancer screening and predict the risk of early stage lung cancer.

Chronic inflammation is well established as a hallmark in lung carcinogenesis [8-10]. Several lines of evidence have revealed that inflammatory biomarkers such as C-reactive protein (CRP) can predict the significant risk of lung cancer [11-16]. For instance, elevated CRP was observed to confer a more than two-fold increased risk of lung cancer [11]. However, most of previous evidence on this subject was based on retrospective studies mainly involving smokers, and whether the resultant association with lung cancer can be extrapolated to non-smokers remains an open question. It is widely recognized that smoking status has a major impact on the molecular pathogenesis of lung cancer [17]. Moreover, the association of inflammatory biomarkers with lung cancer risk was rarely reported in early stage patients, who are clinically valuable to help identify susceptibility biomarkers. To fill this gap in knowledge, we therefore designed a prospective study, seeking to investigate the association between circulating inflammatory biomarkers and the risk for early stage lung adenocarcinoma among 228 patients and 228 matched controls. In addition, $85.96 \%$ of patients were never smokers and $69.74 \%$ were diagnosed at stage IA, which renders us sufficient power in further stratified explorations. In the present study, 10 widely-evaluated inflammatory biomarkers were measured in all study participants, including CRP, interleukin 1 alpha (IL-1a), interleukin 1 beta (IL-1b), interleukin 6 (IL-6), IL-10, interferon-gamma (IFN-r), transforming growth factor alpha (TGF-a), macrophage-derived chemokine (MDC), B lymphocyte chemoattractant (BLC) and monokine induced by gamma interferon (MIG), and they were previously reported to be associated with lung cancer [11-16, 18, 19].

\section{RESULTS}

\section{Baseline characteristics}

This study included 228 patients with early stage lung adenocarcinoma and 228 age-, sex- and smoking-matched controls, and their demographic and clinicopathologic characteristics are presented in Table 1. The mean (standard deviation or SD) age of patients was 58.86 (9.69) years, and $61.40 \%$ of them were female patients $(n=140)$. Never smokers accounted for $85.96 \%$ of patients $(\mathrm{n}=196)$. Of 228 patients, $159(69.74 \%)$ were at stage IA, $19(8.33 \%)$ at stage IB and $50(21.93 \%)$ at stage II.

\section{Inflammatory biomarkers and lung adenocarcinoma risk}

The detection rates of 10 inflammatory biomarkers in circulation are shown in Supplementary Table S1. Notably, the detection rate was $100 \%$ for CRP, BLC, MDC and MIG, respectively.

Four out of 10 inflammatory biomarkers were associated with the risk of early stage lung adenocarcinoma at a significance level of 5\% (Table 2 and Supplementary Table S2). For the comparison of patients in the $4^{\text {th }}$ quartile with the $1^{\text {st }}$ quartile, the multivariate OR $(95 \% \mathrm{CI})$ was 0.29 (0.16-0.53) for MDC $(\mathrm{P}<0.0001), 4.17$ (2.23-7.79) for $\mathrm{BLC}(\mathrm{P}<0.0001), 0.40(0.22-0.74)$ for $\mathrm{MIG}(\mathrm{P}=0.013)$ and $0.58(0.37-0.92)$ for IL-10 $(\mathrm{P}=0.021)$. Two biomarkers, $\mathrm{MDC}$ and BLC, survived the Bonferroni correction for multiple comparisons $(\mathrm{P}<0.05 / 10$, here 10 is the total number of examined biomarkers). Pearson correlation analysis revealed a weak correlation between MDC and BLC ( $\mathrm{r}=-0.022, \mathrm{P}=0.645)$ (Supplementary Table S3). To yield more information, the BLC/MDC ratio was created to examine their prediction for the risk of early stage lung adenocarcinoma, and as expected there was a nearly 10fold increased risk for patients in the $4^{\text {th }}$ quartile relative to patients in the $1^{\text {st }}$ quartile (Table 2).

\section{Stratified analysis of significant inflammatory biomarkers}

There were 196 patients with early stage lung adenocarcinoma and 196 matched controls who were both never smokers. When analysis was restricted to these never smokers, MDC and BLC were still significantly associated with lung adenocarcinoma risk, with the comparison of patients in the $4^{\text {th }}$ quartile with the $1^{\text {st }}$ quartile yielding an OR of 0.37 (95\% CI: $0.21-0.66, \mathrm{P}<0.0001)$ and 2.78 (95\% CI: $1.48-5.22, \mathrm{P}=0.001$ ), respectively (Table 3 ). In addition, significance persisted after restricting analysis to 159 patients with stage IA lung adenocarcinoma and 159 matched controls, that is, elevated levels of MDC and BLC were associated with a $74 \%$ reduced $(\mathrm{OR}=0.26,95 \%$ CI: 0.12-0.50, $\mathrm{P}<0.0001)$ and $110 \%$ increased $(\mathrm{OR}=2.10$, 95\% CI: 1.06-4.00, $\mathrm{P}=0.019)$ risk for stage IA lung adenocarcinoma, respectively (Table 3 ).

\section{Inflammatory biomarkers and subcentimeter lung adenocarcinoma}

As shown in Table 4, BLC was associated with a 2.90-fold (95\% CI: 1.03-8.17, $\mathrm{P}=0.037$ ) increased risk of subcentimeter lung adenocarcinoma (tumor size less than $1 \mathrm{~cm}$ ) for the comparison of patients in the $4^{\text {th }}$ quartile with the $1^{\text {st }}$ quartile. Further ROC curve analysis revealed that BLC was a significant diagnostic biomarker for subcentimeter lung adenocarcinoma, with the area under the curve of 0.63 (95\% CI: 0.54-0.72, $\mathrm{P}=0.0089)$ for BLC (Figure 1). The sensitivity, specificity and positive 
Table 1: Baseline characteristics of early stage lung adenocarcinoma patients and matched controls

\begin{tabular}{|c|c|c|c|}
\hline Characteristics & Patients $(\mathrm{N}=228)$ & Controls $(\mathrm{N}=228)$ & P value \\
\hline Age (years), mean (SD) & $58.86(9.69)$ & $58.82(9.68)$ & 0.970 \\
\hline \multicolumn{4}{|l|}{ Sex, N (\%) } \\
\hline Female & $140(61.40)$ & $140(61.40)$ & 1.000 \\
\hline Male & $88(38.60)$ & $88(38.60)$ & \\
\hline \multicolumn{4}{|l|}{ Smoking status, N (\%) } \\
\hline Ever smokers & $32(14.04)$ & $32(14.04)$ & 1.000 \\
\hline Never smokers & $196(85.96)$ & $196(85.96)$ & \\
\hline \multicolumn{4}{|l|}{ TNM stage } \\
\hline IA & $159(69.74)$ & - & \\
\hline IB & $19(8.33)$ & - & \\
\hline IIA & $19(8.33)$ & - & \\
\hline IIB & $31(13.60)$ & & \\
\hline \multicolumn{4}{|c|}{ Biomarkers (pg/mL), mean (SD) } \\
\hline CRP & $2057507.3(321510.9)$ & $1738483.5(238799.1)$ & 0.230 \\
\hline CXCL13/BLC & $50.8(29.2)$ & $44.4(35.3)$ & 0.072 \\
\hline CCL22 /MDC & $90.0(47.0)$ & $155.7(127.9)$ & $<0.001$ \\
\hline CXCL9/MIG & $27.8(25.7)$ & $35.2(33.3)$ & 0.008 \\
\hline IL-6 & $12.2(7.5)$ & $11.1(6.4)$ & 0.077 \\
\hline
\end{tabular}

Abbreviations: SD, standard deviation; N (\%), number (percentage). P was calculated by the t-test or the Mann-Whitney U test or the Chisq test where appropriate.

likelihood ratio were $0.72,0.52$ and 1.50 , respectively (Supplementary Table S4).

The differences in inflammatory biomarkers among patients with adenocarcinoma in situ (AIS, pure lepidic growth), minimally invasive adenocarcinoma (MIA, predominant lepidic growth with invasion of $5 \mathrm{~mm}$ or less) and invasive adenocarcinoma (IA, predominant lepidic growth with invasion $>5 \mathrm{~mm}$ and other pathologic types of lung adenocarcinoma) showed that there was an increasing trend for BLC with the progression of subcentimeter lung adenocarcinoma from AIS to MIA and IA $(\mathrm{P}=0.046)$, as illustrated in Figure 2 and Supplementary Table S5.

\section{DISCUSSION}

To the best of our knowledge, this is so far the first study that has prospectively evaluated multiple inflammatory biomarkers in circulation and the risk for early stage lung adenocarcinoma in a Chinese population. The key finding of this study was that two inflammatory biomarkers, MDC and BLC, were independently associated with the significant risk of early stage lung adenocarcinoma in overall population as well as in non-smokers and in stage IA patients. Moreover, BLC was found for the first time to have a close link with subcentimeter lung cancer risk, and this biomarker was proposed to play a carcinogenic role in the development and progression of lung adenocarcinoma.

Several large studies have showed that some inflammatory biomarkers could predict the risk of experiencing lung cancer [11-16, 20]. A prospective study by Shiels et al evaluated the relationship between 68 inflammatory biomarkers and lung cancer risk in 526 lung cancer patients and 592 matched controls, and 11 biomarkers were identified to be in significant association with lung cancer [11]. Subsequent replication of this association among the same lung cancer patients and enlarged matched controls $(n=625)$ found that only 4 biomarkers remained statistically significant [20]. Although a large number of previous studies examined the susceptibility of inflammatory biomarkers to lung cancer risk, none have focused on patients with early stage lung cancer, as these patients are difficult to detect but have optimal survival benefits. To fill this void, we measured 10 widely-evaluated inflammatory biomarkers among 228 patients with early stage lung adenocarcinoma and 228 matched healthy controls, and our findings demonstrated 
Table 2: Risk prediction of MDC and BLC for early stage lung adenocarcinoma

\begin{tabular}{lcccc}
\hline \multicolumn{1}{c}{ Biomarkers, pg/mL } & Patients N(\%) & Controls N(\%) & OR (95\% CI), P & $\mathbf{P}_{\text {trend }}$ \\
\hline MDC & & & & \\
$<60.9$ & $72(31.6)$ & $42(18.4)$ & 1 & $<0.0001$ \\
$60.9-93.9$ & $68(29.8)$ & $46(20.2)$ & $0.76(0.43-1.35), 0.360$ & \\
$93.9-140.6$ & $51(22.4)$ & $63(27.6)$ & $0.40(0.22-0.72), 0.002$ & \\
$>140.6$ & $37(16.2)$ & $77(33.8)$ & $0.29(0.16-0.53), 5.7 \times 10^{-5}$ & \\
BLC & & & 1 & $<0.0001$ \\
$<26.4$ & $46(20.2)$ & $69(30.3)$ & & \\
$26.4-39.8$ & $50(21.9)$ & $63(27.6)$ & $1.51(0.86-2.67), 0.154$ & $<0.0001$ \\
$39.8-57.0$ & $58(25.4)$ & $58(25.4)$ & $1.77(1.03-3.03), 0.038$ & \\
$>57.0$ & $74(32.5)$ & $38(16.7)$ & $4.17(2.23-7.79), 8.0 \times 10^{-6}$ & \\
BLC/MDC & & & & \\
1 & $23(10.1)$ & $91(39.9)$ & & \\
2 & $62(27.2)$ & $52(22.8)$ & $5.46(2.55-11.66), 1.2 \times 10^{-5}$ & \\
3 & $69(30.3)$ & $45(19.7)$ & $9.39(4.26-20.72), 2.9 \times 10^{-8}$ & \\
4 & $74(32.5)$ & $40(17.5)$ & $9.98(4.57-21.82), 8.2 \times 10^{-9}$ & \\
\hline
\end{tabular}

Abbreviations: N (\%), number (percentage); OR, odds ration; 95\% CI, 95\% confidence interval. Adjusted for matching variables (age, sex and smoking history), history of chronic bronchitis/emphysema, history of coronary heart disease or heart attack, family history of lung cancer and regular use of aspirin/ibuprofen.

that BLC and MDC were two significant independent predictors for the risk of experiencing early stage lung adenocarcinoma.

BLC, also known as B cell-attracting chemokine 1 (BCA-1) or Chemokine CXC ligand 13 protein (CXCL13), is a member of the CXC subtype of chemokine superfamily.. As evidenced, BLC was involved in the carcinogenesis of several solid tumors, including breast cancer, neuronal cancer and prostatic cancer [21-24]. In addition, elevated BLC levels were observed in both nonsmall cell lung cancer (NSCLC) tissues and sera relative to controls [25]. Extending the results of previous studies, our findings demonstrated that BLC was a remarkable predictive factor for early stage lung adenocarcinoma, and patients in $4^{\text {th }}$ quartile of BLC levels were over 4 times more likely of experiencing early stage lung adenocarcinoma relative to those in $1^{\text {st }}$ quartile, consistent with the findings in the study by Shiels et al [11]. What's more, our findings also indicated a carcinogenic role of BLC in the progression of lung adenocarcinoma from AIS to MIA and IA. The biological mechanisms underlying this role are not fully understood, and further functional characterization is encouraged.

MDC, also known as $\mathrm{C}-\mathrm{C}$ motif chemokine 22 (CCL22), is a member of the $\mathrm{C}-\mathrm{C}$ family of chemokines that are produced by monocyte-derived macrophages and dendritic cells [26]. There is evidence that MDC was up- regulated in some tumor entities, suggesting its oncogenic potentials $[27,28]$. The down-regulation of MDC in turn suggested its tumor suppressive potentials [29]. Nakanishi et al have found that elevated MDC expression was closely linked to longer disease-free survival time and lower risk of lung cancer recurrence after surgical resection [30]. Contrastingly in the study by Shiels et al, MDC was identified as a significant risk factor for lung cancer, that is, patients with elevated MDC levels had a 1.63 -fold increased lung cancer risk in discovery phase and a 2.09-folded increased risk in replication phase [11]. Our findings instead supported a protective role of MDC in lung carcinogenesis. The reasons for this discrepancy are likely due to the study populations of different races (Chinese and Caucasian) and the confounding impact of smoking status between the present study and the study by Shiels et al [11]. In fact, over $90 \%$ of lung cancer patients were smokers in the study by Shiels et al [11], compared to only $14.4 \%$ in the present study.

CRP, a systemic marker of chronic inflammation, has been found correlated with increased lung cancer risk in many previous studies [11-15]. For instance, Chaturvedi et al found that elevated CRP levels are associated with nearly two-fold increased lung cancer risk [12]. However, in the present study, CRP levels did not show significant differences between the case and control subjects. The main cause maybe contributes to the intimate connection 
Table 3: Stratified analysis of MDC and BLC with early stage lung adenocarcinoma

\begin{tabular}{|c|c|c|c|c|c|c|c|c|c|}
\hline \multicolumn{5}{|c|}{ No-smoking lung cancer risk } & \multicolumn{5}{|c|}{ Stage IA lung cancer risk } \\
\hline $\begin{array}{l}\text { Biomarkers, } \\
\mathrm{pg} / \mathrm{mL}\end{array}$ & $\begin{array}{c}\text { Patients } \\
\text { N }(\%)\end{array}$ & $\begin{array}{c}\text { Controls } \\
\text { N }(\%)\end{array}$ & $\begin{array}{c}\text { OR }(95 \% \text { CI }) \\
\text { P }\end{array}$ & $\mathbf{P}_{\text {trend }}$ & $\begin{array}{c}\text { Biomarkers, } \\
\text { pg/mL }\end{array}$ & $\begin{array}{c}\text { Patients } \\
\text { N(\%) }\end{array}$ & $\begin{array}{c}\text { Controls } \\
\mathrm{N}(\%)\end{array}$ & OR $(95 \% \mathrm{CI}), \mathrm{P}$ & $\mathbf{P}_{\text {trend }}$ \\
\hline MDC & & & & & MDC & & & & \\
\hline$<60.2$ & $60(30.6)$ & $37(18.9)$ & 1 & $<0.0001$ & $<60.3$ & $50(31.4)$ & $29(18.2)$ & 1 & $<0.0001$ \\
\hline $60.2-92.1$ & $60(30.6)$ & $39(19.9)$ & $\begin{array}{c}1.00(0.55-1.82), \\
0.990\end{array}$ & & $60.3-91.9$ & $48(30.2)$ & $32(20.1)$ & $\begin{array}{c}0.87(0.45-1.70) \\
0.690\end{array}$ & \\
\hline $92.1-139.2$ & $41(20.9)$ & $57(29.1)$ & $\begin{array}{c}0.48(0.26-0.86) \\
0.014\end{array}$ & & 91.9-134.7 & $37(23.3)$ & $43(27.0)$ & $\begin{array}{c}0.49(0.25-0.98) \\
0.043\end{array}$ & \\
\hline$>139.2$ & $35(17.9)$ & $63(32.1)$ & $\begin{array}{c}0.37(0.21-0.66) \\
0.001\end{array}$ & & $>134.7$ & $24(15.1)$ & $55(34.6)$ & $\begin{array}{c}0.26(0.12-0.50) \\
0.00011\end{array}$ & \\
\hline BLC & & & & & BLC & & & & \\
\hline$<26.4$ & $41(20.9)$ & $56(28.6)$ & 1 & 0.001 & $<27.0$ & $37(23.3)$ & $42(26.4)$ & 1 & 0.019 \\
\hline $26.4-39.7$ & $40(20.5)$ & $54(27.6)$ & $\begin{array}{c}1.05(0.89-1.87), \\
0.870\end{array}$ & & $27.0-39.9$ & $32(20.1)$ & $48(30.2)$ & $\begin{array}{c}0.74(0.52-1.40) \\
0.360\end{array}$ & \\
\hline $39.7-55.7$ & $51(26.0)$ & $51(26.0)$ & $\begin{array}{c}1.60(1.06-2.22), \\
0.230\end{array}$ & & $39.9-56.9$ & $40(25.2)$ & $40(25.2)$ & $\begin{array}{c}1.21(0.76-2.20) \\
0.120\end{array}$ & \\
\hline$>55.7$ & $64(32.0)$ & $35(17.9)$ & $\begin{array}{c}2.78(1.48-5.22), \\
0.001\end{array}$ & & $>56.9$ & $50(31.4)$ & $29(18.2)$ & $\begin{array}{c}2.10(1.06-4.00) \\
0.032\end{array}$ & \\
\hline
\end{tabular}

Abbreviations: N (\%), number (percentage); OR, odds ration; 95\% CI, 95\% confidence interval. Adjusted for matching variables (age, sex and smoking history), history of chronic bronchitis/emphysema, history of coronary heart disease or heart attack, family history of lung cancer and regular use of aspirin/ibuprofen.

Table 4: Risk prediction of MDC and BLC for subcentimetre lung adenocarcinoma

\begin{tabular}{|c|c|c|c|c|}
\hline Biomarkers, pg/mL & Patients N (\%) & Controls N (\%) & OR $(95 \%$ CI $), P$ & $\mathbf{P}_{\text {trend }}$ \\
\hline \multicolumn{5}{|l|}{ MDC } \\
\hline$<60.5$ & $20(28.2)$ & $15(21.1)$ & 1 & 0.058 \\
\hline $60.5-91.7$ & $21(29.6)$ & $15(21.1)$ & $1.09(0.42-2.85), 0.86$ & \\
\hline $91.7-133.2$ & $17(23.9)$ & $19(26.8)$ & $0.68(0.27-1.73), 0.42$ & \\
\hline$>133.2$ & $13(18.3)$ & $22(31.0)$ & $0.37(0.14-1.11), 0.078$ & \\
\hline \multicolumn{5}{|l|}{ BLC } \\
\hline$<26.2$ & $15(21.1)$ & $20(28.2)$ & 1 & 0.037 \\
\hline $26.2-41.8$ & $15(21.1)$ & $22(31.0)$ & $0.90(0.40-2.18), 0.79$ & \\
\hline $41.8-57.0$ & $18(25.4)$ & $17(23.9)$ & $1.50(0.62-3.55), 0.34$ & \\
\hline$>57.0$ & $23(32.4)$ & $12(16.9)$ & $2.90(1.03-8.17), 0.040$ & \\
\hline
\end{tabular}

Abbreviations: N (\%), number (percentage); OR, odds ration; 95\% CI, 95\% confidence interval. Adjusted for matching variables (age, sex and smoking history), history of chronic bronchitis/emphysema, history of coronary heart disease or heart attack, family history of lung cancer and regular use of aspirin/ibuprofen.

between cigarette smoking and evaluated CRP levels [31], since smokers were only accounted for $14.04 \%$ in our study.

It is generally recognized that the diagnosis of subcentimeter lung cancer is a challenging task for oncologists because of its tiny tumor size. Several studies have shown that lung cancer of $1 \mathrm{~cm}$ or less in diameter also has the potentials to spread locally, regionally and systemically [32, 33]. Thus, early detection of subcentimeter lung cancer is still a matter 
of contention. In this study, we interestingly found that patients with elevated BLC levels had nearly three-fold increased subcentimeter lung adenocarcinoma risk, and its sensitivity for diagnosing subcentimeter lung adenocarcinoma reached $72.0 \%$ yet the specificity is not that satisfactory $(52.0 \%)$. Nevertheless, our findings provide a promising inflammatory biomarker for the prediction of subcentimeter lung adenocarcinoma, and we agree that further efforts are needed to combine this inflammatory biomarker with $\mathrm{CT}$ detection for the differential diagnosis of subcentimeter lung cancer as a whole.

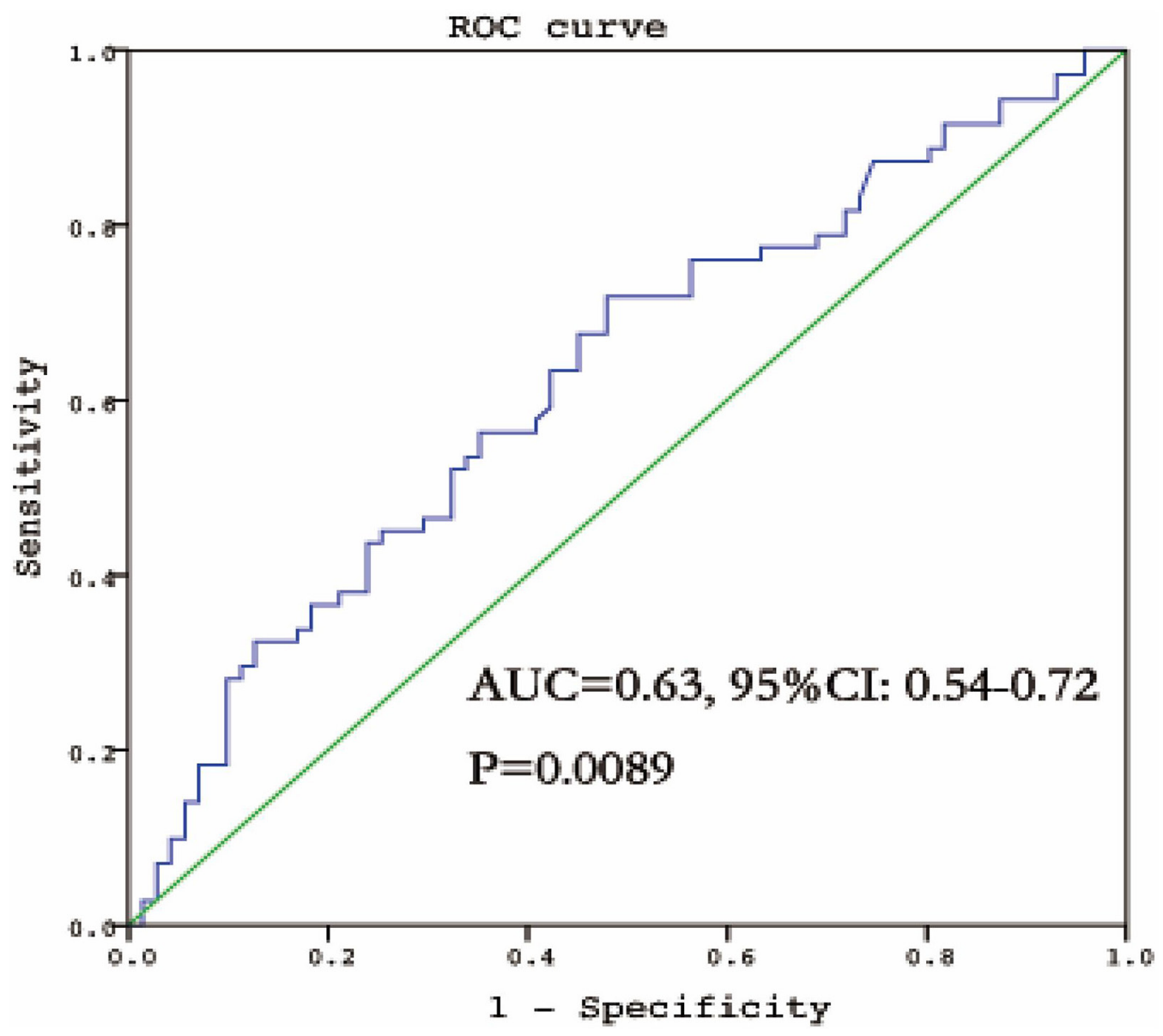

Figure 1: The ROC curve of BLC for the diagnosis of subcentimeter lung adenocarcinoma. ROC, receiver operating characteristic; AUC, area under curve.

A

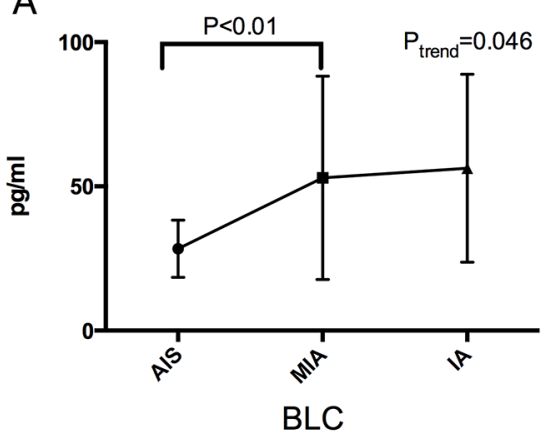

B

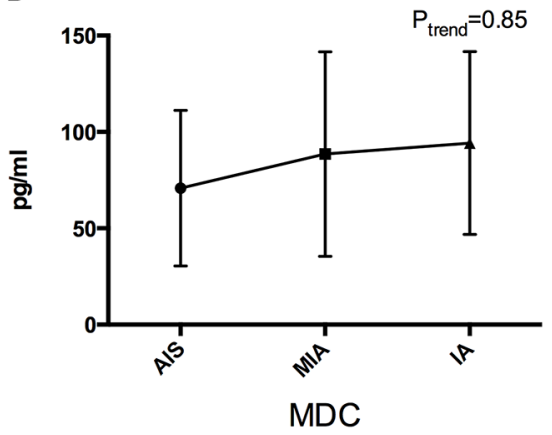

C

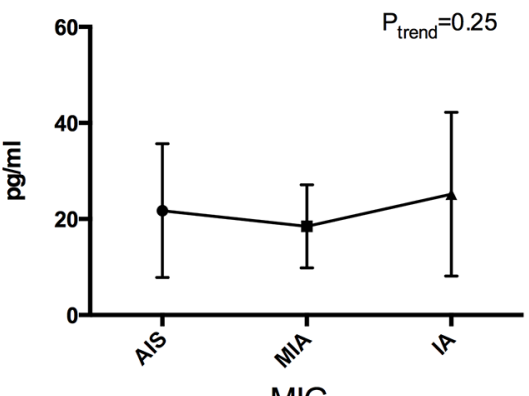

MIG

Figure 2: The different levels of BLC A. MDC B. and MIG C. among patients with AIS, MIA and IA. BLC, B lymphocyte chemoattractant, MDC, Macrophage-Derived Chemokine; MIG, Monokine Induced by Gamma Interferon; AIS, adenocarcinoma in situ; MIA, minimally invasive adenocarcinoma; IA, invasive adenocarcinoma. 
Our study is not without limitations. On the one hand, our findings need to be replicated in another independent population to further confirm the predictive role of MDC and BLC in susceptibility to early stage lung adenocarcinoma. On the other hand, only lung andenocarcinoma patients were enrolled in the present study, and future studies with large samples specifically designed to examine the association of the two inflammatory biomarkers with lung squamous carcinoma and the other types of NSCLC are warranted.

In conclusion, our findings for the first time demonstrate that two inflammatory biomarkers, MDC and BLC, were independently associated with the significant risk of early stage lung adenocarcinoma, and this significant association persisted even in non-smokers and in stage IA patients. Moreover, BLC could be a promising biomarker for accessorily diagnosing subcentimeter lung cancer and may play a carcinogenic role in the progression of lung adenocarcinoma. For practical reasons, our findings may provide the basis for future personalized medicine whereby early stage lung cancer patients with abnormal inflammatory profiles can be detected earlier and receive timely therapeutic interventions.

\section{MATERIALS AND METHODS}

\section{Study population}

Lung cancer patients who were histologically confirmed according to the World Health Organization classification criteria [34] received lung resection in the Shanghai Chest Hospital between September 2013 and March 2015. The inclusion criteria of cases were: 1) patients with early stage (IA to IIB) lung adenocarcinoma; 2) available blood samples before lung resection. Reasons for exclusion were missing data on smoking history or family cancer history or missing consent for etiologic

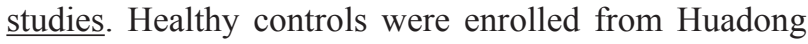
Sanatorium, and one control subject was selected for each patient matched on age ( \pm 2 year), sex and smoking history. All study participants gave written informed consent before interview procedures and biospecimen collection. The conduct of this study was approved by the Institutional Review Boards of local hospitals.

From each patient, information was recorded at the time of enrollment, including age, sex, smoking history, family cancer history, medical history and working history. Pathologic stage was determined by the International Association for the Study of Lung Cancer (IASLC) TNM (tumor-node-metastasis) classification, $8^{\text {th }}$ edition [35].

\section{Inflammatory biomarkers}

Preoperative blood samples were collected in coded heparinized tubes, and they were centrifuged at 2400-3000 rpm for 15 minutes at room temperature. Serum samples were frozen within 2 hours of collection and stored at $-80^{\circ} \mathrm{C}$ until assayed.

On the basis of the results of previous studies $[11,15,20]$, a total of 10 inflammatory biomarkers with potential implications in lung carcinogenesis were selected for measurement using the Luminex bead-based assay. Concentrations of 10 inflammatory biomarkers in circulation were calculated using a four or five parameter standard curve. All serum samples were assayed in duplicate and the results were averaged for analysis. Samples of both patients and matched controls were assayed on the same batch, and each patient-control pair was plated into adjacent wells. Within each batch, one pair of blinded duplicates and a pooled serum sample across batches were assayed to evaluate the reproducibility and drift across batches.

\section{Statistical analysis}

Data were analyzed by the SPSS software version 20.0 (SPSS Inc., Chicago, IL, USA). Statistical significance was taken as two-sided $\mathrm{P}<0.05$, and multiple testing was controlled by the Bonferroni correction $(\mathrm{P}<$ 0.05 / 10 biomarkers).

For inflammatory biomarkers, measurements below the lowest limit of detection (LLOD) were assigned a value of half the LLOD as previously described [11]. All biomarkers were categorized into groups based on the proportion of individuals with measurements more than the LLOD as follows: biomarkers with more than $75 \%$ of individuals over the LLOD were categorized into quartiles; biomarkers with $50 \%$ to $75 \%$ of individuals over the LLOD were categorized into tertiles; biomarkers with $25 \%$ to $50 \%$ of individuals over the LLOD were categorized as below and above the median; and biomarkers with $10 \%$ to $25 \%$ of individuals over the LLOD were categorized as the undetectable and the detectable.

Conditional Logistic regression was used to quantify the association of inflammatory biomarkers with lung cancer risk, and effect-size estimates were expressed as odds ratios (ORs) and 95\% confidence intervals (CIs). Besides the matched variables (age, sex and smoking history), adjustment also included history of chronic bronchitis/emphysema, history of coronary heart disease or heart attack, family history of lung cancer and regular use of aspirin/ibuprofen. In addition, the association of inflammatory biomarkers with lung cancer risk in non-smokers, in stage IA patients and in patients with subcentimeter tumor was separately analyzed. The relationship of inflammatory biomarkers was assessed by Pearson correlation analysis. Receiver operating characteristic (ROC) curve was created to evaluate the role of inflammatory biomarkers in diagnosing subcentimeter lung cancer. One-way ANOVA was used to compare inflammatory biomarkers among patients with adenocarcinoma in situ (AIS), minimally invasive adenocarcinoma (MIA) and invasive adenocarcinoma (IA). 


\section{CONFLICTS OF INTEREST}

The authors have no conflicts of interest to declare.

\section{FUNDING}

This work was supported by the National Science Foundation of China (NSFC) (grant no. 81572249), Chinese Society of Clinical Oncology fund (grant no. Y-S2014-003), and Shanghai Municipal Commission of Health and Family Planning (grant no. 2013ZYJB0402).

\section{REFERENCES}

1. Siegel RL, Miller KD and Jemal A. Cancer statistics, 2015. CA: a cancer journal for clinicians. 2015; 65:5-29.

2. Lortet-Tieulent J, Soerjomataram I, Ferlay J, Rutherford $\mathrm{M}$, Weiderpass $\mathrm{E}$ and Bray F. International trends in lung cancer incidence by histological subtype: adenocarcinoma stabilizing in men but still increasing in women. Lung cancer. 2014; 84:13-22.

3. Murakami S, Ito H, Tsubokawa N, Mimae T, Sasada S, Yoshiya T, Miyata Y, Yokose T, Okada M and Nakayama H. Prognostic value of the new IASLC/ATS/ERS classification of clinical stage IA lung adenocarcinoma. Lung cancer. 2015; 90:199-204.

4. Wang T, Nelson RA, Bogardus A and Grannis FW, Jr. Fiveyear lung cancer survival: which advanced stage nonsmall cell lung cancer patients attain long-term survival? Cancer. 2010; 116:1518-1525.

5. National Lung Screening Trial Research T, Church TR, Black WC, Aberle DR, Berg CD, Clingan KL, Duan F, Fagerstrom RM, Gareen IF, Gierada DS, Jones GC, Mahon I, Marcus PM, Sicks JD, Jain A and Baum S. Results of initial low-dose computed tomographic screening for lung cancer. The New England journal of medicine. 2013; 368:1980-1991.

6. Humphrey LL, Deffebach M, Pappas M, Baumann C, Artis K, Mitchell JP, Zakher B, Fu R and Slatore CG. Screening for lung cancer with low-dose computed tomography: a systematic review to update the US Preventive services task force recommendation. Annals of internal medicine. 2013; 159:411-420.

7. Gopal M, Abdullah SE, Grady JJ and Goodwin JS. Screening for lung cancer with low-dose computed tomography: a systematic review and meta-analysis of the baseline findings of randomized controlled trials. Journal of thoracic oncology 2010; 5:1233-1239.

8. Houghton AM, Mouded M and Shapiro SD. Common origins of lung cancer and COPD. Nature medicine. 2008; 14:1023-1024.

9. Punturieri A, Szabo E, Croxton TL, Shapiro SD and Dubinett SM. Lung cancer and chronic obstructive pulmonary disease: needs and opportunities for integrated research. Journal of the National Cancer Institute. 2009; 101:554-559.

10. O'Callaghan DS, O'Donnell D, O'Connell F and O'Byrne KJ. The role of inflammation in the pathogenesis of nonsmall cell lung cancer. Journal of thoracic oncology 2010; 5:2024-2036.

11. Shiels MS, Pfeiffer RM, Hildesheim A, Engels EA, Kemp TJ, Park JH, Katki HA, Koshiol J, Shelton G, Caporaso NE, Pinto LA and Chaturvedi AK. Circulating inflammation markers and prospective risk for lung cancer. Journal of the National Cancer Institute. 2013; 105:1871-1880.

12. Chaturvedi AK, Caporaso NE, Katki HA, Wong HL, Chatterjee N, Pine SR, Chanock SJ, Goedert JJ and Engels EA. C-reactive protein and risk of lung cancer. Journal of clinical oncology 2010; 28:2719-2726.

13. Il'yasova D, Colbert LH, Harris TB, Newman AB, Bauer DC, Satterfield S and Kritchevsky SB. Circulating levels of inflammatory markers and cancer risk in the health aging and body composition cohort. Cancer epidemiology, biomarkers \& prevention 2005; 14:2413-2418.

14. Allin KH, Bojesen SE and Nordestgaard BG. Baseline $\mathrm{C}$-reactive protein is associated with incident cancer and survival in patients with cancer. Journal of clinical oncology 2009; 27:2217-2224.

15. Pine SR, Mechanic LE, Enewold L, Chaturvedi AK, Katki HA, Zheng YL, Bowman ED, Engels EA, Caporaso NE and Harris CC. Increased levels of circulating interleukin 6 , interleukin 8, C-reactive protein, and risk of lung cancer. Journal of the National Cancer Institute. 2011; 103:1112-1122.

16. Bigbee WL, Gopalakrishnan V, Weissfeld JL, Wilson DO, Dacic S, Lokshin AE and Siegfried JM. A multiplexed serum biomarker immunoassay panel discriminates clinical lung cancer patients from high-risk individuals found to be cancer-free by CT screening. Journal of thoracic oncology 2012; 7:698-708.

17. Lan Q, Hsiung CA, Matsuo K, Hong YC, Seow A, Wang Z, Hosgood HD, 3rd, Chen K, Wang JC, Chatterjee N, Hu W, Wong MP, Zheng W, et al. Genome-wide association analysis identifies new lung cancer susceptibility loci in never-smoking women in Asia. Nature genetics. 2012; 44:1330-1335.

18. Lan $\mathrm{X}$, Lan $\mathrm{T}$ and Faxiang Q. Interleukin-10 promoter polymorphism and susceptibility to lung cancer: a systematic review and meta-analysis. International journal of clinical and experimental medicine. 2015; $8: 15317-15328$.

19. Kiyohara C, Horiuchi T, Takayama K and Nakanishi Y. IL1B rs1143634 polymorphism, cigarette smoking, alcohol use, and lung cancer risk in a Japanese population. Journal of thoracic oncology 2010; 5:299-304.

20. Shiels MS, Katki HA, Hildesheim A, Pfeiffer RM, Engels EA, Williams M, Kemp TJ, Caporaso NE, Pinto LA and 
Chaturvedi AK. Circulating Inflammation Markers, Risk of Lung Cancer, and Utility for Risk Stratification. Journal of the National Cancer Institute. 2015; 107.

21. Panse J, Friedrichs K, Marx A, Hildebrandt Y, Luetkens T, Barrels K, Horn C, Stahl T, Cao Y, Milde-Langosch K, Niendorf A, Kroger N, Wenzel S, et al. Chemokine CXCL13 is overexpressed in the tumour tissue and in the peripheral blood of breast cancer patients. British journal of cancer. 2008; 99:930-938.

22. Del Grosso F, Coco S, Scaruffi P, Stigliani S, Valdora F, Benelli R, Salvi S, Boccardo S, Truini M, Croce M, Ferrini S, Longo L and Tonini GP. Role of CXCL13-CXCR5 crosstalk between malignant neuroblastoma cells and Schwannian stromal cells in neuroblastic tumors. Molecular cancer research : 2011; 9:815-823.

23. El Haibi CP, Sharma PK, Singh R, Johnson PR, Suttles J, Singh S and Lillard JW, Jr. PI3Kp110-, Src-, FAKdependent and DOCK2-independent migration and invasion of CXCL13-stimulated prostate cancer cells. Molecular cancer. 2010; 9:85.

24. El-Haibi CP, Singh R, Sharma PK, Singh S and Lillard JW, Jr. CXCL13 mediates prostate cancer cell proliferation through JNK signalling and invasion through ERK activation. Cell proliferation. 2011; 44:311-319.

25. Singh R, Gupta P, Kloecker GH, Singh S and Lillard JW, Jr. Expression and clinical significance of CXCR5/CXCL13 in human nonsmall cell lung carcinoma. International journal of oncology. 2014; 45:2232-2240.

26. Yamashita U and Kuroda E. Regulation of macrophagederived chemokine (MDC, CCL22) production. Critical reviews in immunology. 2002; 22:105-114.

27. Narita D, Seclaman E, Anghel A, Ilina R, Cireap N, Negru S, Sirbu IO, Ursoniu S and Marian C. Altered levels of plasma chemokines in breast cancer and their association with clinical and pathological characteristics. Neoplasma. 2016; 63:141-149.

28. Jafarzadeh A, Fooladseresht H, Minaee K, Bazrafshani MR, Khosravimashizi A, Nemati M, Mohammadizadeh M, Mohammadi MM and Ghaderi A. Higher circulating levels of chemokine CCL22 in patients with breast cancer: evaluation of the influences of tumor stage and chemokine gene polymorphism. Tumour biology 2015; 36:1163-1171.
29. Zhou M, Bracci PM, McCoy LS, Hsuang G, Wiemels JL, Rice T, Zheng S, Kelsey KT, Wrensch MR and Wiencke JK. Serum macrophage-derived chemokine/CCL22 levels are associated with glioma risk, CD4 T cell lymphopenia and survival time. International journal of cancer Journal international du cancer. 2015; 137:826-836.

30. Nakanishi $T$, Imaizumi $K$, Hasegawa $Y$, Kawabe $T$, Hashimoto N, Okamoto M and Shimokata K. Expression of macrophage-derived chemokine (MDC)/CCL22 in human lung cancer. Cancer immunology, immunotherapy : 2006; 55:1320-1329.

31. Shiels MS, Katki HA, Freedman ND, Purdue MP, Wentzensen N, Trabert B, Kitahara CM, Furr M, Li Y, Kemp TJ, Goedert JJ, Chang CM, Engels EA, et al. Cigarette smoking and variations in systemic immune and inflammation markers. Journal of the National Cancer Institute. 2014; 106.

32. Asamura H, Suzuki K, Watanabe S, Matsuno Y, Maeshima A and Tsuchiya R. A clinicopathological study of resected subcentimeter lung cancers: a favorable prognosis for ground glass opacity lesions. The Annals of thoracic surgery. 2003; 76:1016-1022.

33. Okada M, Nishio W, Sakamoto T, Uchino K, Yuki T, Nakagawa A and Tsubota N. Effect of tumor size on prognosis in patients with non-small cell lung cancer: the role of segmentectomy as a type of lesser resection. The Journal of thoracic and cardiovascular surgery. 2005; 129:87-93.

34. Travis WD, Brambilla E, Nicholson AG, Yatabe Y, Austin JH, Beasley MB, Chirieac LR, Dacic S, Duhig E, Flieder DB, Geisinger K, Hirsch FR, Ishikawa Y, et al. The 2015 World Health Organization Classification of Lung Tumors: Impact of Genetic, Clinical and Radiologic Advances Since the 2004 Classification. Journal of thoracic oncology 2015; 10:1243-1260.

35. Rami-Porta R, Bolejack V, Crowley J, Ball D, Kim J, Lyons G, Rice T, Suzuki K, Thomas CF, Jr., Travis WD, $\mathrm{Wu}$ YL, Staging I, Prognostic Factors Committee AB and Participating I. The IASLC Lung Cancer Staging Project: Proposals for the Revisions of the T Descriptors in the Forthcoming Eighth Edition of the TNM Classification for Lung Cancer. Journal of thoracic oncology 2015; 10:990-1003. 